\title{
NTUA-SLP at IEST 2018: Ensemble of Neural Transfer Methods for Implicit Emotion Classification
}

\author{
Alexandra Chronopoulou ${ }^{1 *}$, Aikaterini Margatina ${ }^{1 *}$ \\ Christos Baziotis $^{1,2}$, Alexandros Potamianos ${ }^{1,3}$ \\ ${ }^{1}$ School of ECE, National Technical University of Athens, Athens, Greece \\ ${ }^{2}$ Department of Informatics, Athens University of Economics and Business, Athens, Greece \\ ${ }^{3}$ Signal Analysis and Interpretation Laboratory (SAIL), USC, Los Angeles, USA \\ el12068@central.ntua.gr, el12108@central.ntua.gr \\ cbaziotis@mail.ntua.gr, potamecentral.ntua.gr
}

\begin{abstract}
In this paper we present our approach to tackle the Implicit Emotion Shared Task (IEST) organized as part of WASSA 2018 at EMNLP 2018. Given a tweet, from which a certain word has been removed, we are asked to predict the emotion of the missing word. In this work, we experiment with neural Transfer Learning (TL) methods. Our models are based on LSTM networks, augmented with a selfattention mechanism. We use the weights of various pretrained models, for initializing specific layers of our networks. We leverage a big collection of unlabeled Twitter messages, for pretraining word2vec word embeddings and a set of diverse language models. Moreover, we utilize a sentiment analysis dataset for pretraining a model, which encodes emotion related information. The submitted model consists of an ensemble of the aforementioned TL models. Our team ranked $3^{\text {rd }}$ out of 30 participants, achieving an $F_{1}$ score of 0.703 .
\end{abstract}

\section{Introduction}

Social media, especially micro-blogging services like Twitter, have attracted lots of attention from the NLP community. The language used is constantly evolving by incorporating new syntactic and semantic constructs, such as emojis or hashtags, abbreviations and slang, making natural language processing in this domain even more demanding. Moreover, the analysis of such content leverages the high availability of datasets offered from Twitter, satisfying the need for large amounts of data for training.

\footnotetext{
*These authors contributed equally to this work.
}

Emotion recognition is particularly interesting in social media, as it has useful applications in numerous tasks, such as public opinion detection about political tendencies (Pla and Hurtado, 2014; Tumasjan et al., 2010; $\mathrm{Li}$ and $\mathrm{Xu}, 2014$ ), stock market monitoring (Si et al., 2013; Bollen et al., 2011b), tracking product perception (Chamlertwat et al., 2012), even detection of suicide-related communication (Burnap et al., 2015).

In the past, emotion analysis, like most NLP tasks, was tackled by traditional methods that included hand-crafted features or features from sentiment lexicons (Nielsen, 2011; Mohammad and Turney, 2010, 2013; Go et al., 2009) which were fed to classifiers such as Naive Bayes and SVMs (Bollen et al., 2011a; Mohammad et al., 2013; Kiritchenko et al., 2014). However, deep neural networks achieve increased performance compared to traditional methods, due to their ability to learn more abstract features from large amounts of data, producing state-of-the-art results in emotion recognition and sentiment analysis (Deriu et al., 2016; Goel et al., 2017; Baziotis et al., 2017).

In this paper, we present our work submitted to the WASSA 2018 IEST (Klinger et al., 2018). In the given task, the word that triggers emotion is removed from each tweet and is replaced by the token [\#TARGETWORD\# ]. The objective is to predict its emotion category among 6 classes: anger, disgust, fear, joy, sadness and surprise. Our proposed model employs 3 different TL schemes of pretrained models: word embeddings, a sentiment model and language models. 


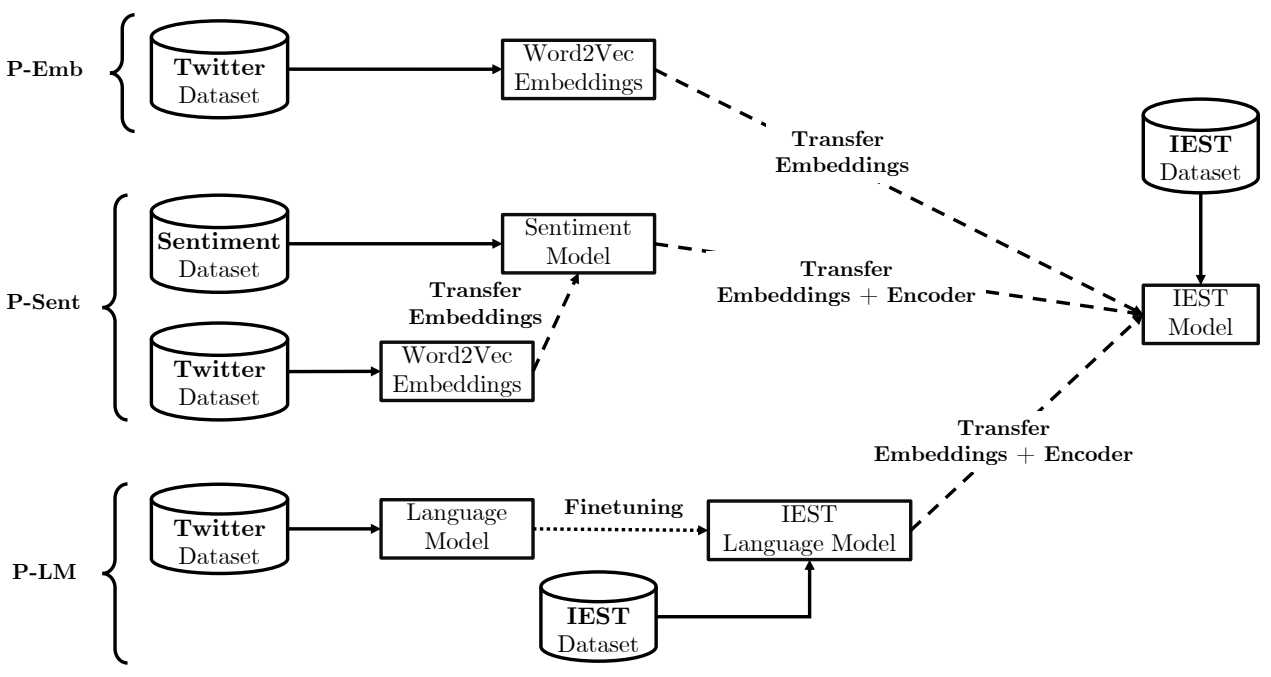

Figure 1: High-level overview of our TL approaches.

\section{Overview}

Our approach is composed of the following three steps: (1) pretraining, in which we train word2vec word embeddings (P-Emb), a sentiment model (PSent) and Twitter-specific language models ( $\mathrm{P}$ LM), (2) transfer learning, in which we transfer the weights of the aforementioned models to specific layers of our IEST classifier and (3) ensembling, in which we combine the predictions of each TL model. Figure 1 depicts a high-level overview of our approach.

\subsection{Data}

Apart from the IEST dataset, we employ a Se$\mathrm{mEval}$ dataset for sentiment classification and other manually-collected unlabeled corpora for our language models.

Unlabeled Twitter Corpora. We collected a dataset of 550 million archived English Twitter messages, from 2014 to 2017 . This dataset is used for calculating word statistics for our text preprocessing pipeline and training our word2vec word embeddings presented in Sec. 4.1.

For training our language models, described in Sec. 4.3, we sampled three subsets of this corpus. The first consists of $2 \mathrm{M}$ tweets, all of which contain emotion words. To create the dataset, we selected tweets that included one of the six emotion classes of our task (anger, disgust, fear, joy, sadness and surprise) or synonyms. We ensured that this dataset is balanced by concatenating approximately $350 \mathrm{~K}$ tweets from each category. The second chunk has $5 \mathrm{M}$ tweets, randomly selected from the initial 550M corpus. We aimed to create a general sub-corpus, so as to focus on the structural relationships of words, instead of their emotional content. The third chunk is composed of the two aforementioned corpora. We concatenated the $2 \mathrm{M}$ emotion dataset with $2 \mathrm{M}$ generic tweets, creating a final $4 \mathrm{M}$ dataset. We denote the three corpora as EmoCorpus (2M), EmoCorpus+ (4M) and GenCorpus (5M).

Sentiment Analysis Dataset. We use the dataset of SemEval17 Task4A (Sent17) (Rosenthal et al., 2017) for training our sentiment classifier as described in Sec. 4.2. The dataset consists of Twitter messages annotated with their sentiment polarity (positive, negative, neutral). The training set contains $56 \mathrm{~K}$ tweets and the validation set $6 \mathrm{~K}$ tweets.

\subsection{Preprocessing}

To preprocess the tweets, we use Ekphrasis (Baziotis et al., 2017), a tool geared towards text from social networks, such as Twitter and Facebook. Ekphrasis performs Twitter-specific tokenization, spell correction, word normalization, segmentation (for splitting hashtags) and annotation.

\subsection{Word Embeddings}

Word embeddings are dense vector representations of words which capture semantic and syntactic information. For this reason, we employ the word2vec (Mikolov et al., 2013) algorithm to train our word vectors, as described in Sec. 4.1.

\subsection{Transfer Learning}

Transfer Learning (TL) uses knowledge from a learned task so as to improve the performance of 
a related task by reducing the required training data (Torrey and Shavlik, 2010; Pan et al., 2010). In computer vision, transfer learning is employed in order to overcome the deficit of training samples for some categories by adapting classifiers trained for other categories (Oquab et al., 2014). With the power of deep supervised learning, learned knowledge can even be transferred to a totally different task (i.e. ImageNet (Krizhevsky et al., 2012)).

Following this logic, TL methods have also been applied to NLP. Pretrained word vectors (Mikolov et al., 2013; Pennington et al., 2014) have become standard components of most architectures. Recently, approaches that leverage pretrained language models have emerged, which learn the compositionality of language, capture long-term dependencies and context-dependent features. For instance, ELMo contextual word representations (Peters et al., 2018) and ULMFiT (Howard and Ruder, 2018) achieve state-of-the-art results on a wide variety of NLP tasks. Our work is mainly inspired by ULMFiT, which we extend to the Twitter domain.

\subsection{Ensembling}

We combine the predictions of our 3 TL schemes with the intent of increasing the generalization ability of the final classifier. To this end, we employ a pretrained word embeddings approach, as well as a pretrained sentiment model and a pretrained LM. We use two ensemble schemes, namely unweighted average and majority voting.

Unweighted Average (UA). In this approach, the final prediction is estimated from the unweighted average of the posterior probabilities for all different models. Formally, the final prediction $p$ for a training instance is estimated by:

$$
p=\underset{c}{\arg \max } \frac{1}{C} \sum_{i=1}^{M} \overrightarrow{p_{i}}, \quad p_{i} \in \mathbb{R}^{C}
$$

where $C$ is the number of classes, $M$ is the number of different models, $c \in\{1, \ldots, C\}$ denotes one class and $\overrightarrow{p_{i}}$ is the probability vector calculated by model $i \in\{1, \ldots, M\}$ using softmax function.

Majority Voting (MV). Majority voting approach counts the votes of all different models and chooses the class with most votes. Compared to UA, MV is affected less by single-network decisions. However, this schema does not consider any information derived from the minority models. Formally, for a task with $C$ classes and $M$ different models, the prediction for a specific instance is estimated as follows:

$$
\begin{aligned}
& v_{c}=\sum_{i=1}^{M} F_{i}(c) \\
& p=\underset{c \in\{1, \ldots, C\}}{\arg \max } v_{c}
\end{aligned}
$$

where $v_{c}$ denotes the votes for class $c$ from all different models, $F_{i}$ is the decision of the $i^{\text {th }}$ model, which is either 1 or 0 with respect to whether the model has classified the instance in class $c$ or not and $p$ is the final prediction.

\section{Network Architecture}

All of our TL schemes share the same architecture: A 2-layer LSTM with a self-attention mechanism. It is shown in Figure 2.

Embedding Layer. The input to the network is a Twitter message, treated as a sequence of words. We use an embedding layer to project the words $w_{1}, w_{2}, \ldots, w_{N}$ to a low-dimensional vector space $R^{W}$, where $W$ is the size of the embedding layer and $N$ the number of words in a tweet.

LSTM Layer. An LSTM takes as input a sequence of word embeddings and produces word annotations $h_{1}, h_{2}, \ldots, h_{N}$, where $h_{i}$ is the hidden state at time-step $i$, summarizing all the information of the sentence up to $w_{i}$. We use bidirectional LSTM to get word annotations that summarize the information from both directions. A bi-LSTM consists of a forward $\vec{f}$ that parses the sentence from $w_{1}$ to $w_{N}$ and a backward $\overleftarrow{f}$ that parses it from $w_{N}$ to $w_{1}$. We obtain the final annotation for each word $h_{i}$, by concatenating the annotations from both directions, $h_{i}=\overrightarrow{h_{i}} \| \overleftarrow{h_{i}}, \quad h_{i} \in R^{2 L}$, where $\|$ denotes the concatenation operation and $L$ the size of each LSTM. When the network is initialized with pretrained LMs, we employ unidirectional instead of bi-LSTMs.

Attention Layer. To amplify the contribution of the most informative words, we augment our LSTM with an attention mechanism, which assigns a weight $a_{i}$ to each word annotation $h_{i}$. We compute the fixed representation $r$ of the whole input message, as the weighted sum of all the word annotations.

$$
\begin{aligned}
& e_{i}=\tanh \left(W_{h} h_{i}+b_{h}\right), \quad e_{i} \in[-1,1] \\
& a_{i}=\frac{\exp \left(e_{i}\right)}{\sum_{t=1}^{T} \exp \left(e_{t}\right)}, \quad \sum_{i=1}^{T} a_{i}=1
\end{aligned}
$$




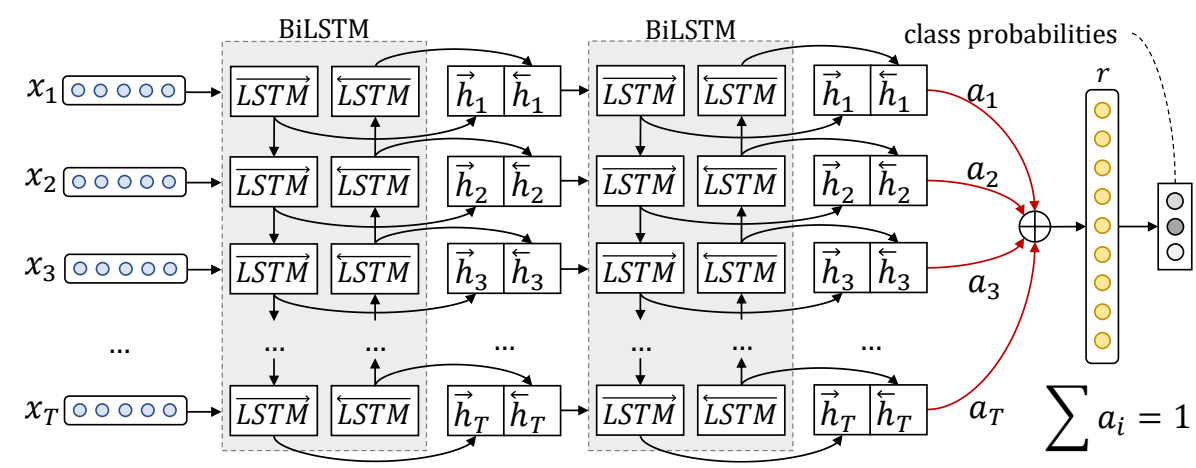

Figure 2: The proposed model, composed of a 2-layer bi-LSTM with a deep self-attention mechanism. When the model is initialized with pretrained LMs, we use unidirectional LSTM instead of bidirectional.

$$
r=\sum_{i=1}^{T} a_{i} h_{i}, \quad r \in R^{2 L}
$$

where $W_{h}$ and $b_{h}$ are the attention layer's weights. Output Layer. We use the representation $r$ as feature vector for classification and we feed it to a fully-connected softmax layer with $L$ neurons, which outputs a probability distribution over all classes $p_{c}$ as described in Eq. 6:

$$
p_{c}=\frac{e^{W r+b}}{\sum_{i \in[1, L]}\left(e^{W_{i} r+b_{i}}\right)}
$$

where $W$ and $b$ are the layer's weights and biases.

\section{Transfer Learning Approaches}

\subsection{Pretrained Word Embeddings (P-Emb)}

In the first approach, we train word2vec word embeddings with which we initialize the embedding layer of our network. The weights of the embedding layer remain frozen during training. The word $2 v e c$ word embeddings are trained on the 550M Twitter corpus (Sec. 2.1), with negative sampling of 5 and minimum word count of 20, using Gensim's ( ̌̌ehưřek and Sojka, 2010) implementation. The resulting vocabulary contains 800,000 words.

\subsection{Pretrained Sentiment Model (P-Sent)}

In the second approach, we first train a sentiment analysis model on the Sent17 dataset, using the architecture described in Sec. 3. The embedding layer of the network is initialized with our pretrained word embeddings. Then, we fine-tune the network on the IEST task, by replacing its last layer with a task-specific layer.

\subsection{Pretrained Language Model (P-LM)}

The third approach consists of the following steps: (1) we first train a language model on a generic Twitter corpus, (2) we fine-tune the LM on the task at hand and finally, (3) we transfer the embedding and RNN layers of the LM, we add attention and output layers and fine-tune the model on the target task.

LM Pretraining. We collect three Twitter datasets as described in Sec. 2.1 and for each one we train an LM. In each dataset we use the 50,000 most frequent words as our vocabulary. Since the literature concerning LM transfer learning is limited, especially in the Twitter domain, we aim to explore the desired characteristics of the pretrained LM. To this end, our contribution in this research area lies in experimenting with a taskrelevant corpus (EmoCorpus), a generic one (GenCorpus) and a mixture of both (EmoCorpus+).

LM Fine-tuning. This step is crucial since, albeit the diversity of the general-domain data used for pretraining, the data of the target task will likely have a different distribution.

We thus fine-tune the three pretrained LMs on the IEST dataset, employing two approaches. The first is simple fine-tuning, according to which all layers of the model are trained simultaneously. The second one is a simplified yet similar approach to gradual unfreezing, proposed in (Howard and Ruder, 2018), which we denote as Simplified Gradual Unfreezing (SGU). According to this method, after we have transfered the pretrained embedding and LSTM weights, we let only the output layer fine-tune for $n-1$ epochs. At the $n^{t h}$ epoch, we unfreeze both LSTM layers. We let the model fine-tune, until epoch $k-1$. Finally, at epoch $k$, we also unfreeze the embed- 
ding layer and let the network train until convergence. In other words, we experiment with pairs of numbers of epochs, $\{\mathrm{n}, \mathrm{k}\}$, where $n$ denotes the epoch when we unfreeze the LSTM layers and $k$ the epoch when we unfreeze the embedding layer. Naive fine-tuning poses the risk of catastrophic forgetting, or else abruptly losing the knowledge of a previously learnt task, as information relevant to the current task is incorporated. Therefore, to prevent this from happening, we unfreeze the model starting from the last layer, which is taskspecific, and after some epochs we progressively unfreeze the next, more general layers, until all layers are unfrozen.

LM Transfer. This is the final step of our TL approach. We now have several LMs from the second step of the procedure. We transfer their embedding and RNN weights to a final target classifier. We again experiment with both simple and more sophisticated fine-tuning techniques, to find out which one is more helpful to this task.

Furthermore, we introduce the concatenation method which was inspired by the correlation of language modeling and the task at hand. We use pretrained LMs to leverage the fact that the task is basically a cloze test. In an LM, the probability of occurrence of each word, is conditioned on the preceding context, $P\left(w_{t} \mid w_{1}, \ldots, w_{t-1}\right)$. In RNNbased LMs, this probability is encoded in the hidden state of the RNN, $P\left(w_{t} \mid h_{t-1}\right)$. To this end, we concatenate the hidden state of the LSTM, right before the missing word, $h_{\text {implicit }}$, to the output of the self-attention mechanism, $r$ :

$$
r^{\prime}=r \| h_{\text {implicit }}, \quad h_{i} \in R^{2 L}
$$

where $L$ is the size of each LSTM, and then feed it to the output linear layer. This way, we preserve the information which implicitly encodes the probability of the missing word.

\section{Experiments and Results}

\subsection{Experimental Setup}

Training. We use Adam algorithm (Kingma and $\mathrm{Ba}, 2014)$ to optimize our networks, with minibatches of size 64 and clip the norm of the gradients (Pascanu et al., 2013) at 0.5, as an extra safety measure against exploding gradients. We also used PyTorch (Paszke et al., 2017) and Scikitlearn (Pedregosa et al., 2011).

Hyperparameters. For all our models, we employ the same 2-layer attention-based LSTM ar- chitecture (Sec. 3). All the hyperparameters used are shown in Table 1.

\begin{tabular}{l|r|r|r}
\hline Layer & P-Emb & P-Sent & P-LM \\
\hline Embedding & 300 & 300 & 400 \\
\hline Embedding noise & 0.1 & 0.1 & 0.1 \\
\hline Embedding dropout & 0.2 & 0.2 & 0.2 \\
\hline LSTM size & 400 & 400 & $600 / 800$ \\
\hline LSTM dropout & 0.4 & 0.4 & 0.4 \\
\hline
\end{tabular}

Table 1: Hyper-parameters of our models.

\subsection{Official Results}

Our team ranked $3^{\text {rd }}$ out of 30 participants, achieving $0.703 \mathrm{~F} 1$-score on the official test set. Table 2 shows the official ranking of the top scoring teams.

\begin{tabular}{c|c|c}
\hline Rank & Team Name & Macro F1 \\
\hline 1 & Amobee & 0.714 \\
\hline 2 & IIIDYT & 0.710 \\
\hline $\mathbf{3}$ & NTUA-SLP & $\mathbf{0 . 7 0 3}$ \\
\hline 4 & UBC-NLP & 0.693 \\
\hline 5 & Sentylic & 0.692 \\
\hline
\end{tabular}

Table 2: Results of the WASSA IEST competition.

\subsection{Experiments}

Baselines. In Table 5 we compare the proposed TL approaches against two strong baselines: (1) a Bag-of-Words (BoW) model with TF-IDF weighting and (2) a Bag-of-Embeddings (BoE) model, where we retrieve the word2vec representations of the words in a tweet and compute the tweet representation as the centroid of the constituent word2vec representations. Both $B o W$ and $B o E$ features are then fed to a linear SVM classifier, with tuned $C=0.6$. All of our reported F1-scores are calculated on the evaluation (dev) set, due to time constraints.

$\boldsymbol{P}$-Emb and $\boldsymbol{P}$-Sent models $(4.1,4.2)$. We evaluate the $P$-Emb and $P$-Sent models, using both bidirectional and unidirectional LSTMs. The F1 score of our best models is shown in Table 5. As expected, bi-LSTM models achieve higher performance.

$\boldsymbol{P}$ - $\boldsymbol{L M}$ (4.3). For the experiments with the pretrained LMs, we intend to transfer not just the first layer of our network, but rather the whole model, so as to capture more high-level features of language. As mentioned above, there are three distinct steps concerning the training procedure of this TL approach: (1) LM pretraining: we train three LMs on the EmoCorpus, EmoCorpus+ and 


\begin{tabular}{c|ccc|r}
\hline LM Fine-tuning & \multicolumn{3}{|c|}{ LM Transfer } & \\
\hline & Simple FT & SGU & Concat. & F1 \\
\hline \multirow{4}{*}{ Simple FT } & $\checkmark$ & & & 0.672 \\
& $\checkmark$ & & $\checkmark$ & 0.667 \\
& & $\checkmark$ & & 0.676 \\
& & $\checkmark$ & $\checkmark$ & 0.673 \\
\hline \multirow{3}{*}{ SGU } & $\checkmark$ & & & 0.673 \\
& $\checkmark$ & & $\checkmark$ & 0.667 \\
& & $\checkmark$ & & 0.678 \\
& & $\checkmark$ & $\checkmark$ & $\mathbf{0 . 6 8 2}$ \\
\hline
\end{tabular}

Table 3: Results of the P-LM, trained on the EmoCorpus. The first column refers to the way we finetune each LM on the IEST dataset and the second to the way we finally fine-tune the classifier on the same dataset.

\begin{tabular}{l|r}
\hline Dataset & F1 \\
\hline EmoCorpus & 0.682 \\
\hline EmoCorpus+ & 0.680 \\
\hline GenCorpus & 0.675 \\
\hline
\end{tabular}

Table 4: Comparison of the P-LM models, all finetuned with $S G U$ and Concat. methods.

GenCorpus corpora, (2) LM fine-tuning: we finetune the LMs on the IEST dataset, with 2 different ways. The first one is simple fine-tuning, while the second one is our simplified gradual unfreezing (SGU) technique. (3) LM transfer: We now have $6 \mathrm{LMs}$, fine-tuned on the IEST dataset. We transfer their weights to our final emotion classifier, we add attention to the LSTM layers and we experiment again with our 2 ways of fine-tuning and the concatenation method proposed in Sec. 4.3.

In Table 3 we present all possible combinations of transferring the $P-L M$ to the IEST task. We observe that SGU consistently outperforms Simple Fine-Tuning (Simple FT). Due to the difficulty in running experiments for all possible combinations, we compare our best approach, namely $S G U$ + Concat., with $P-L M$ s trained on our three unlabeled Twitter corpora, as depicted in Table 4. Even though EmoCorpus contains less training examples, $P-L M$ s trained on it learn to encode more useful information for the task at hand.

\subsection{Ensembling}

Our submitted model is an ensemble of the models with the best performance. More specifically, we leverage the following models: (1) TL of pretrained word embeddings, (2) TL of pretrained sentiment classifier, (3) TL of 3 different LMs, trained on $2 \mathrm{M}, 4 \mathrm{M}$ and $5 \mathrm{M}$ respectively. We use Unweighted Average (UA) ensembling of our best

\begin{tabular}{l|c}
\hline Model & $\mathrm{F} 1$ \\
\hline Bag of Words (BoW) & 0.601 \\
\hline Bag of Embeddings (BoE) & 0.605 \\
\hline \hline P-Emb & 0.668 \\
\hline P-Sent & 0.671 \\
\hline P-LM & $\mathbf{0 . 6 7 5}$ \\
\hline \hline P-Emb + bidir. & 0.684 \\
\hline P-Sent + bidir. & 0.674 \\
\hline P-LM + SGU & 0.679 \\
\hline P-LM + SGU + Concat. & 0.682 \\
\hline \hline Ensembling (UA) P-Emb + P-Sent & 0.684 \\
\hline Ensembling (UA) P-Sent + P-LM & 0.695 \\
\hline Ensembling (UA) P-Emb + P-LM & 0.701 \\
\hline \hline Ensembling (MV) All & 0.700 \\
\hline Ensembling (UA) All & $\mathbf{0 . 7 0 2}$ \\
\hline
\end{tabular}

Table 5: Results of our experiments when tested on the evaluation (dev) set. $B o W$ and $B o E$ are our baselines, while $P$-Emb, $P$-Sent and $P$-LM our proposed TL approaches. $S G U$ stands for Simplified Gradual Unfreezing, bidir. for bi-LSTM, Concat. for the concatenation method, UA for Unweighted Average and $M V$ for Majority Voting ensembling.

models from all aforementioned approaches. Our final results on the evaluation data are shown in Table 5.

\subsection{Discussion}

As shown in Table 5, we observe that all of our proposed models achieve individually better performance than our baselines by a large margin. Moreover, we notice that, when the three models are trained with unidirectional LSTM and the same number of parameters, the $P-L M$ outperforms both the $P$-Emb and the P-Sent models. As expected, the upgrade to bi-LSTM improves the results of $P$-Emb and $P$-Sent. We hypothesize that $P-L M$ with bidirectional pretrained language models would have outperformed both of them. Furthermore, we conclude that both SGU for finetuning and the concatenation method enhance the performance of the $P$ - $L M$ approach. As far as the ensembling is concerned, both approaches, $M V$ and $U A$, yield similar performance improvement over the individual models. In particular, we notice that adding the $P$ - $L M$ predictions to the ensemble contributes the most. This indicates that $P$ $L M s$ encode more diverse information compared to the other approaches. 


\section{Conclusion}

In this paper we describe our deep-learning methods for missing emotion words classification, in the Twitter domain. We achieved very competitive results in the IEST competition, ranking $3^{\text {rd }} / 30$ teams. The proposed approach is based on an ensemble of Transfer Learning techniques. We demonstrate that the use of refined, high-level features of text, as the ones encoded in language models, yields a higher performance. In the future, we aim to experiment with subword-level models, as they have shown to consistently face the OOV words problem (Sennrich et al., 2015; Bojanowski et al., 2016), which is more evident in Twitter. Moreover, we would like to explore other transfer learning approaches.

Finally, we share the source code of our models ${ }^{1}$, in order to make our results reproducible and facilitate further experimentation in the field.

\section{References}

Christos Baziotis, Nikos Pelekis, and Christos Doulkeridis. 2017. Datastories at semeval-2017 task 4: Deep lstm with attention for message-level and topic-based sentiment analysis. In Proceedings of the 11th International Workshop on Semantic Evaluation (SemEval-2017), pages 747-754.

Piotr Bojanowski, Edouard Grave, Armand Joulin, and Tomas Mikolov. 2016. Enriching word vectors with subword information. arXiv preprint arXiv:1607.04606.

Johan Bollen, Huina Mao, and Alberto Pepe. 2011a. Modeling public mood and emotion: Twitter sentiment and socio-economic phenomena. Icwsm, $11: 450-453$.

Johan Bollen, Huina Mao, and Xiaojun Zeng. 2011b. Twitter mood predicts the stock market. Journal of computational science, 2(1):1-8.

Pete Burnap, Walter Colombo, and Jonathan Scourfield. 2015. Machine classification and analysis of suicide-related communication on twitter. In Proceedings of the 26th ACM conference on hypertext \& social media, pages 75-84. ACM.

Wilas Chamlertwat, Pattarasinee Bhattarakosol, Tippakorn Rungkasiri, and Choochart Haruechaiyasak. 2012. Discovering consumer insight from twitter via sentiment analysis. J. UCS, 18(8):973-992.

Jan Deriu, Maurice Gonzenbach, Fatih Uzdilli, Aurelien Lucchi, Valeria De Luca, and Martin Jaggi.

\footnotetext{
1/github.com/alexandra-chron/ wassa-2018
}

2016. Swisscheese at semeval-2016 task 4: Sentiment classification using an ensemble of convolutional neural networks with distant supervision. In Proceedings of the 10th international workshop on semantic evaluation, EPFL-CONF-229234, pages 1124-1128.

Alec Go, Richa Bhayani, and Lei Huang. 2009. Twitter sentiment classification using distant supervision. CS224N Project Report, Stanford, 1(12).

Pranav Goel, Devang Kulshreshtha, Prayas Jain, and Kaushal Kumar Shukla. 2017. Prayas at emoint 2017: An ensemble of deep neural architectures for emotion intensity prediction in tweets. In Proceedings of the 8th Workshop on Computational Approaches to Subjectivity, Sentiment and Social Media Analysis, pages 58-65.

Jeremy Howard and Sebastian Ruder. 2018. Finetuned language models for text classification. CoRR, abs/1801.06146.

Diederik Kingma and Jimmy Ba. 2014. Adam: A method for stochastic optimization. arXiv preprint arXiv:1412.6980.

Svetlana Kiritchenko, Xiaodan Zhu, and Saif M. Mohammad. 2014. Sentiment analysis of short informal texts. Journal of Artificial Intelligence Research, 50:723-762.

Roman Klinger, Orphée de Clercq, Saif M. Mohammad, and Alexandra Balahur. 2018. Iest: Wassa2018 implicit emotions shared task. In Proceedings of the 9th Workshop on Computational Approaches to Subjectivity, Sentiment and Social Media Analysis, Brussels, Belgium. Association for Computational Linguistics.

Alex Krizhevsky, Ilya Sutskever, and Geoffrey E Hinton. 2012. Imagenet classification with deep convolutional neural networks. In Advances in neural information processing systems, pages 1097-1105.

Weiyuan Li and Hua Xu. 2014. Text-based emotion classification using emotion cause extraction. Expert Systems with Applications, 41(4):1742-1749.

Tomas Mikolov, Ilya Sutskever, Kai Chen, Greg S. Corrado, and Jeff Dean. 2013. Distributed representations of words and phrases and their compositionality. In Advances in Neural Information Processing Systems, pages 3111-3119.

Saif M. Mohammad, Svetlana Kiritchenko, and Xiaodan Zhu. 2013. NRC-Canada: Building the stateof-the-art in sentiment analysis of tweets. arXiv preprint arXiv:1308.6242.

Saif M Mohammad and Peter D Turney. 2010. Emotions evoked by common words and phrases: Using mechanical turk to create an emotion lexicon. In Proceedings of the NAACL HLT 2010 workshop on computational approaches to analysis and generation of emotion in text, pages 26-34. Association for Computational Linguistics. 
Saif M Mohammad and Peter D Turney. 2013. Crowdsourcing a word-emotion association lexicon. Computational Intelligence, 29(3):436-465.

Finn Årup Nielsen. 2011. A new anew: Evaluation of a word list for sentiment analysis in microblogs. arXiv preprint arXiv:1103.2903.

Maxime Oquab, Leon Bottou, Ivan Laptev, and Josef Sivic. 2014. Learning and transferring mid-level image representations using convolutional neural networks. In Proceedings of the IEEE conference on computer vision and pattern recognition, pages 1717-1724.

Sinno Jialin Pan, Qiang Yang, et al. 2010. A survey on transfer learning. IEEE Transactions on knowledge and data engineering, 22(10):1345-1359.

Razvan Pascanu, Tomas Mikolov, and Yoshua Bengio. 2013. On the difficulty of training recurrent neural networks. ICML (3), 28:1310-1318.

Adam Paszke, Sam Gross, Soumith Chintala, Gregory Chanan, Edward Yang, Zachary DeVito, Zeming Lin, Alban Desmaison, Luca Antiga, and Adam Lerer. 2017. Automatic differentiation in pytorch.

Fabian Pedregosa, Gaël Varoquaux, Alexandre Gramfort, Vincent Michel, Bertrand Thirion, Olivier Grisel, Mathieu Blondel, Peter Prettenhofer, Ron Weiss, Vincent Dubourg, and others. 2011. Scikitlearn: Machine learning in Python. Journal of Machine Learning Research, 12(Oct):2825-2830.

Jeffrey Pennington, Richard Socher, and Christopher D. Manning. 2014. Glove: Global Vectors for Word Representation. In $E M N L P$, volume 14, pages 1532-1543.

Matthew E Peters, Mark Neumann, Mohit Iyyer, Matt Gardner, Christopher Clark, Kenton Lee, and Luke Zettlemoyer. 2018. Deep contextualized word representations. arXiv preprint arXiv:1802.05365.

Ferran Pla and Lluís-F Hurtado. 2014. Political tendency identification in twitter using sentiment analysis techniques. In Proceedings of COLING 2014, the 25th international conference on computational linguistics: Technical Papers, pages 183-192.

Radim Řehůřrek and Petr Sojka. 2010. Software Framework for Topic Modelling with Large Corpora. In Proceedings of the LREC 2010 Workshop on New Challenges for NLP Frameworks, pages 45-50, Valletta, Malta. ELRA. http://is.muni.cz/publication/ $884893 /$ en

Sara Rosenthal, Noura Farra, and Preslav Nakov. 2017. Semeval-2017 task 4: Sentiment analysis in twitter. In Proceedings of the 11th International Workshop on Semantic Evaluation (SemEval-2017), pages 502-518.
Rico Sennrich, Barry Haddow, and Alexandra Birch. 2015. Neural machine translation of rare words with subword units. arXiv preprint arXiv:1508.07909.

Jianfeng Si, Arjun Mukherjee, Bing Liu, Qing Li, Huayi Li, and Xiaotie Deng. 2013. Exploiting topic based twitter sentiment for stock prediction. In Proceedings of the 51st Annual Meeting of the Association for Computational Linguistics (Volume 2: Short Papers), volume 2, pages 24-29.

Lisa Torrey and Jude Shavlik. 2010. Transfer learning. In Handbook of Research on Machine Learning Applications and Trends: Algorithms, Methods, and Techniques, pages 242-264. IGI Global.

Andranik Tumasjan, Timm Oliver Sprenger, Philipp G Sandner, and Isabell M Welpe. 2010. Predicting elections with twitter: What 140 characters reveal about political sentiment. Icwsm, 10(1):178-185. 